\title{
Index
}

accommodation $34,35,36,42,43,46$, $60,81,87,111,128,131,136$, $138-9,140,150,157,159-60$, 182-3, 196-7, 200, 201, 205-6, 209, 212, 214, 238, 252-3, 256 see also spatial organisation adult education $25,42,148,165,198$, 202, 206-7, 209, 210, 213, 247, 250

adventure see primitivism afforestation 26-7, 57, 80, 130, 138, 150, 197-8, 260

Alden, Percy 14, 26-7

alternative communities 82-7, 203-13

Clousden Hill 83-4

Daisy colony 86

Norton 81,84

Purleigh 85

Whiteway 85-6

see also Grith Fyrd

Amery, Leo 102, 106

An Cor Déantais see Construction Corps

anarchism 82-7, 89, 232

Anstey, Edgar 157, 159, 164

Australia 16, 102, 104-5, 109, 113-14, $116,177-80,189$

Balfour, Gerald 43

Balfour Declaration 211

Barnett, Samuel 16, 38, 39

Besant, Annie 47

Betterton, Henry 149, 152, 177, 253

Beveridge, William 22-3, 40, 47, 48

Birmingham 67, 174, 185

Board of Control 67, 68-70

Bodelschwingh, Friedrich von 19-21, $32,35,59,62$

bodily appearance $5-6,17,44,49,50$,
$65,86,143,154,166-7,183-5$, 190, 199, 202, 240, 249, 250, 255,257

Boer Wars 5, 15, 16, 17, 21, 57, 100

Bondfield, Margaret 45, 90-1, 107, 125-30, 132-5, 142-3, 176, $180,187-8,210,226-7,236$

Booth, Charles 10, 22-3, 32

Booth, William 24, 99

Bourdieu, Pierre 6

Bowers, F. G. 130-1

Brandon Training Centre see Instructional Centres

British Union of Fascists 232

British Women's Emigration Association 105, 174-5

British Women's Temperance Association 62

Brooks, J. L. 14, 34, 36, 234

Brynmawr 196, 204

Burns, John 20, 24-5, 43-6

Cambridge University 197, 199, 200-3

Canada 101, 103-5, 108-10, 113-14, $116,246,255$

Carlisle, Wilson 18, 33, 88

Carlyle, Thomas 9

Carpenter, Edward 78, 83

Catholics 26, 35, 42, 164, 247, 248

Catholic Emigration Society 115

Central Committee for Women's

Training and Employment 127, $129,152,175-6,186,188-9$

Central (Unemployed) Body for London 42-3, 50, 105, 106

Cérésole, Pierre 196, 208

Chamberlain, Neville 149

charity $10,16,20,23,26,32-3,38,45$, $57,100,103,113-14,174,201$ 
Charity Organisation Society 6, 20, 33

Chartist movement 9-10, 13, 78

Children's Fresh Air Mission 16, 103

Christian Union for Social Service $20,33,37,50,51,61,77,81$, 104-5

Church Army 18, 33, 105, 179

Church of Scotland 15, 33, 47, 112-13, $115,116-17,186,256$

Churchill, Winston 67, 110, 112

citizenship 11, 13, 16, 50, 59, 68, 70, 91-3, 133, 208, 231, 247, 249, 260-1

Civilian Conservation Corps 4, 207, 225, 246-7

Claydon Training Centre 107-8, 131 see also Instructional Centres

cleanliness 34, 49, 50, 110, 137-8, 183, 185, 186

Cleveland Work Camps 205-6

Clynes, J. R. 45, 129, 133

coal-mining industry 106, 109, 110, 128-9, 131, 198, 200-1, 229, 230,231

Cole, G. D. H. 117, 135-6

Committee on Empire Migration 117-18

Communist Party of Great Britain 154, 195, 222, 224-7, 230-2, 238-9, 256

community 197, 199-200, 203, 207, 213-14, 247, 252, 254-5, 260-1

concentration camps 3-4, 229, 232-3, 238-9, 249

Congregationalists $14,16,20,33,103$, 125,129

conservatives $9,40,44,112,129,155$, 189

Construction Corps 245, 250-1, 255

Cossar, Dr George 113-15, 117

Crooks, Will 39, 40, 50, 88, 101

Cunningham, Ben 87-90

David Eder Farm 200, 211-13

Davidson, J. C. C. 155, 166

Davison, Ronald C. 154, 156, 210

Deutsche Freischar 204, 207

Dickens, Charles 174

diet 48, 49, 81, 143, 158-9, 204, 209, 213, 234-5, 236, 237

discipline $26,35-6,63-4,71-2,82$, $108,112,161-2,164-6,234$, 238-9 domestic service $60,61,71,93,105$, $115-16,174-5,182-3,184-5$, 187-90, 226

Down, Dr Reginald Langdon 66-7

Down's Syndrome 67

drunkenness $18,26,34,35,42,61-4$, $70,71,82,84,88,204$

Eady, Wilfred 109, 152, 155-6

Edinburgh 11, 46, 65-6, 68, 181, 201

emigration 4, 18, 23, 33, 42, 79, 99-124, 129, 173-8, 211-13, 224, 250

Empire Settlement Act 99, 101, 102, 105-7, 109, 113-15, 118-19

English Home Colonisation Society $13-14,81$

epilepsy 19, 59-61, 69, 258

see also Labour colonies, Belmont Industrial Colony; Chalfont St Peters village colony; David Lewis colony; Horton; Lingfield; Starnthwaite

eugenics $57-9,67-8,70-1,102,118$, 206, 210

ex-servicemen and women 70, 81, 102, 106, 107-8, 175, 259

Fabian Society 14-15, 16, 25, 27, 47, 94

family life 42, 115, 154, 200-1, 226, 232

farm colonies see Labour colonies

Fascism see Nazism

'feeble-minded' 58-9, 66-70, 226, 234-5

see also Labour colonies, Hildenborough; Larbert; Meanwood Park; Redmires

Fels, Joseph 15, 40-1, 90

film 112, 157, 159, 164, 184, 202, 210

Forestry Commission 111, 130-1, 150, 260

Foucault, Michel 71-2, 215

France 20, 21, 196

Freiwilliger Arbeitsdienst 206, 228, 247-8, 253, 255

Gardiner, Rolf 204-8, 246, 250

Geddes, Patrick 78, 90

Germany 16, 18, 19-21, 23, 33, 156, 189, 202, 204, 227-8, 239-40, 247-50, 255 
Girton College see Cambridge University

Glasgow 115, 128, 152, 185, 223-4, 227,235

Glasgow Social Union 19, 21

Goffman, E. 251-2

Government Training Centres 133, 135-7, 139, 143, 167

Wallsend GTC 150

Grith Fyrd 204, 208-11, 246

Guides 201

Haggard, Henry Rider 34, 48, 89-90, 100-2, 113

Hannington, Wal 126, 222, 226, 227 , $228,232,237,239$

Hardie, Keir 12, 39, 44, 101

Hazell, Walter 16, 33, 37-8, 103

Home Training Centres

Appleton Hall, Warrington 181, 182

Beeches, Leamington Spa 179-81

Elms, Market Harborough 173, 178-81, 182, 183, 187

Harden, Newcastle 179-81, 183

Lapsewood, London 181, 183, 187

Millersneuk, Paisley 179-81, 182, 187,188

Waldernheath, Harrogate 181, 182

Hoyland, Jack 196, 199, 222

Hunt, William Henry 35-6, 91

Idleness see stigma

Independent Labour Party 178, 227, 230

Industrial Transference Board 110, 114, 127,128

Inebriates Acts 61-3

Instructional centres

Allerston 150, 158

Ardentinny/Glenfinart 153, 158, $163,235-6$

Blackpool 128, 131, 142

Bourne 111, 116, 236

Brandon 108, 127, 131, 137, 151, $158,226-7$

Brechfa 153, 229, 237

Carshalton 131, 142, 236

Carstairs 111, 116, 142, 150, 230-1, 236

Claydon 142

Cranwich 111, 116, 142

Culford 153
Fermyn Woods 111, 116, 128, 131, 148, 236, 238

Glenbranter 150-1, 158, 162, 255

Glentress 153, 237

Gwydwr 153

Haldon 153

Hamsterley 150, 153, 157, 159, 229, 237

High Lodge 111, 142

Kershopefoot 165, 166, 238

Kielder 153, 163

Knapdale 153, 166

Langdale End 150

Pickworth 159

Poole 131

Presteigne 111, 116, 128, 131, 142

Rendlesham 153

Shobdon 111, 116, 142, 157

Swanton Novers 111, 116

West Tofts 111, 142

International Labour Organisation 245

International Voluntary Service 196-7, 200, 213-14, 233, 246

Ireland 14, 16, 26, 250-1, 255

see also Northern Ireland

Isle of Man 140

Jewish refugees 187, 212, 213

Jones, Gareth 249

Jones, G. S. 10

Jones, Thomas 152

Kenworthy, John Coleman 83, 85

Kropotkin, Pyotr 14, 82-4, 212

Labour colonies

Belmont Industrial Colony 45, 61, 106-7, 128, 224-6, 230, 232, 234, 235

Brogborough 114

Chalfont St Peters village colony 60

Chat Moss 45

Cornton Vale 47, 105, 113, 115, 116-17

Craigielinn 113-14, 115, 117

David Lewis colony 60

Duxhurst Farm Colony 62-3

Fambridge 18, 43

Girgenti House 63-4

Hadleigh 17, 32, 33-8, 39-40, 49, $63,100,104,115,225$

Hairmyres 66

Hildenborough 68 
Hollesley Bay 18, 41-3, 44, 47, 48-9, 105, 106, 128, 152, 223, 224,234

Horton 60

Laindon 40-1, 45, 50, 128, 225

Langdon 69, 71

Langho 64

Langley 33, 103

Larbert 69

Letchworth Garden City 43, 48

Libury Hall 158

Lingfield 6-7, 14, 33, 35-7, 49-50, 61, 63, 70, 234, 258

Meanwood Park 68

Midlocharwood 33, 35-7, 48, 49, 64, 70, 105

Murieston 46

Newdigate 33-4, 63

Ockenden 44

Osea Island 40, 43, 61, 258

Palacerigg 46, 48, 49, 128, 152, 235

Polton 66

Redmires 70

Starnthwaite 17, 60, 79-82

Wallingford 48, 50-1, 225, 259

Washburn Valley 45

Women's Training Colony 90-3

Woodbridge 43

labour exchanges 25-7, 43, 108, 109, $126,130,134,136-7,153-4$, $176,180,184-5,199,223,226$, 229

Labour Party 129, 130, 188, 224-5, 229, 231

see also Independent Labour Party

land settlement 4, 9-10, 12-15, 27, 33, $39,44,45,46,77-87,101-2$, $129,149,206,208$

Mayland 18, 223

Totley 77-8

Lansbury, George 12-13, 39-41, 44, $47,90-1,132,223,225$

Lawrence, Susan 126, 176, 188

laziness see stigma

leadership see management

Leeds 45, 68, 87, 238

leisure 41, 42-3, 108, 112, 139, 163-5, 183, 205, 206, 209, 213, 253, 255-6

see also sport

Local Government Board 40, 43-5, 79,128
Loch, Charles 19, 20, 23, 60

London 10, 12, 22, 25, 33, 38, 39-42, 106,224

London County Council 60, 128, 225,230

Plaistow 87-90

Long, Walter 40, 43

MacDonald, Ramsay 44, 125, 142, 149,228

Maclean, John 223-4

Mallon, James J. 210

management $69,77,84,111-12$, 160-3, 166, 199-200, 203, 206-7, 259-60

Manchester 45, 60, 87, 211

Markham, Violet 152, 166-7, 176, $177-81,186,260-1$

Marshall, Alfred 11-12, 14, 22

masculinity $10,15,50-1,65,103,133$, 207, 248-50, 253-4

Mavor, Professor James 21, 104

Meath, Earl 16, 57, 59

Medical treatment 49, 58-60, 65-6, 137-8, 154-5, 161, 183, 184-6, 202

Mental Deficiency Act 67-8

mental health see 'feeble-minded'

Middlesbrough 154, 195

militarism 111, 136, 159, 160, 165, $205,230,247,248,254-5$

Mills, Herbert Vincent 11-12, 14, 24, 77, 79-82

Milner, Alfred 102, 105, 106

Ministry of Health 68, 106, 128, 185-6

Ministry of Labour 107, 109-12, 116, $117,127,130-1,136,140-2$, $149,162-3,176,180,184,189$, 198, 232, 236, 237, 238-9, 250, 251, 256-8, 259

National deterioration debate see physical deterioration

National Unemployed Workers' Movement 126, 138, 222-9, 231-3, 235-7, 239

nationalism 14, 101, 117, 159, 198, 230, 204-13, 248-9

see also Scottish nationalism

Nazism 189, 202, 206-8, 227-8, 229 , 231-3, 239-40, 248-50, 253

New Zealand 104, 121, 225

Northern Ireland 108-9, 141, 156, 195 
nudity $86,204,208$

Oversea Settlement Board 118

Oversea Settlement Committee 129, 177-9

Oversea Settlement Department 105-6, $114,177-81$

Owenites 4, 9, 11

Oxford University 197, 198

pacifism $85,196,206,208,209,233$, 246

Passmore, John R. 116, 117, 132, 141, $150,151,155,160$

Paton, John Brown 13, 15, 16, 19

Phillips, Dr Marion 176, 180, 187, 226

physical deterioration $10,15-17,26$, $57,99,114,128,143,255$

physical training $16,17,26,57,135$, 149, 157-8, 202, 208, 248

see also sport

placement in work $34,116,139,142$, $149,166-7,174-5,187,247$, 251

'Plaistow landgrabbers' see Triangle Camp

Poor Law 2, 10-11, 12, 14, 20-1, 24, 38-47, 41, 80, 99, 103, 105, $128,149,151,224,228$

Poplar 12-13, 39, 40, 45, 234

primitivism 136, 209-10, 223-4, 253-4 privacy $42,81,159,183$

prostitution 47, 90-3, 174, 226

protest $81,87-90,139-40,224-40$

public relations $112,155,165,184-5$, 188-9, 210, 239

public works 44, 116, 132, 149, 247, 248,250

see also relief works

punishment see discipline

Quakers 4, 196, 210, 259

Quelch, Harry 223

race $14,16,46,65,99,101-2,103$, $106,109,112-13,177,178$, 206-7, 232

'reconditioning' 116, 127-8, 131-3, $142,150,151-2,157,167,199$, 251,260

recruitment 33-4, 41, 103-4, 109-10, 116, 129, 130, 135, 137, 140, $150,153-6$
Compulsory 132-6, 140, 149, 153-4, 155-7, 186, 227, 250

Durham women's camps 201

Grith Fyrd 210-1

Home Training Centres 180, 183, 184-7

UCUC 198-9

Women's Training Colony 91

Reichsarbeitsdienst 156, 247, 248-50, 253

relief work 25, 43, 46, 167, 235, 237

religious services $35,42,164,165$

Royal Commission on the Poor Laws $11,23,25-6,43,46-7,59$

Royal Commission on Unemployment Insurance 134, 135, 149, 155, $181,186,227$

Ruskin, John 14, 77-8, 103

Salvation Army 17, 32, 33, 38, 63, 100, 104, 114-15, 177

Scottish Labour Colony Association 25, $33,35,37,64,105$

Scottish nationalists $112-13,223-4$, 232

Scouts 115, 199

Self-Help Emigration Society 33, 103-4

Service civil international see International Voluntary Service

sexuality $61,86,93,165,214,257$

Shaw, George Bernard 83, 88, 94

Sheffield 11, 70, 77, 91, 199, 226

Shinwell, Manny 129-30

Sims-Williams, M. 197, 199, 214

Smart, Bolton 18, 41-2, 48-9, 234

Society for the Overseas Settlement of British Women 177, 179

St Albans Hostel 179

social service $117,195,196-7,199$, 200-1, 207, 210, 250, 259-60

see also Christian Union for Social Service

social work 27, 33, 35-7, 92, 259

South Africa 106, 109

Soviet Union 224, 225, 240, 255

spatial organisation $18,35,42,58,59$, $60,62,67,69-71,92,159-60$, 214, 252

see also total institutions

Special Areas Act 151

Sport 16, 36, 60, 108, 135, 139, 149, $157,159,163-5,198,201-2$, 205, 209, 248, 254 
Stacey, Enid 81

Stevenson, Daniel 19, 21, 33

stigma 13, 14, 17-18, 21, 23, 24, 26, $27,39,40,47,58,80,87-9$, $126,128,130,133,143,223$, 240, 259-60

strikes 10, 24, 44, 140, 224, 229, 234-8

students 48, 196, 197-203, 204-5, 207, 214, 222, 256, 259

suffrage movement $69,91-3,125,178$, 188

Sutter, Julie 16, 19, 62

'testing camps' $18,108,110,111-12$, $116,127-8,142$

therapeutic communities 259

Thomas, Jimmy H. 107, 112, 127, 132

Tolstoy, Nikolai 85-7

total institutions 251-2

trade unions $43,44,130,134-5$, 142-3, 195, 205-6, 229, 230-1, 237

training, vocational 16, 17, 18, 20, 23, $26,33,36,37,38,39,44,47$, 68-70, 91, 93, 99, 102, 103, $105,108-10,112,115,117$, $127,128-31,132,135,138-9$, $141,148,151,161,162-3$, 173-90, 211, 223, 226, 227, 230-1, 259

see also Government Training Centres

Transfer Instructional Centres 127-8, 130-2, 133-5, 140-2

Triangle Camp 87-90

Tuberculosis 49, 65-6, 70, 91, 154, 184, 235, 259

see also Labour colonies, Hairmyres; Polton

Turnor, Christopher 101, 102, 117

unemployable, the $13,22-3,26-7$, 130, 143

Unemployed Workmen Act 25, 42, 43, 101

Unemployment 2, 4, 10-1, 20-1, 22-4, $25,39-40,87-90,127,143$, 197,211

Unemployment Assistance Act
151-2, 228-9, 236-7

Unemployment Assistance Board 152-3, 231

Unemployment insurance 27, 43, $126,127,129,134,148,149$, 153, 226, 228, 247

see also Royal Commission on Unemployment Insurance

United States of America 16, 104, 246-7, 255

Universities Council for Unemployed Camps 198-203, 214

vagrancy $13,17-18,19,21,23,26,40$, $41,47,80,149$

voluntary action $1,20,25,32,35,37$, $47-8,63,68,90-1,99-102$, 106, 113-15, 116, 173-4, 177, $179,195,199,208,213-15$, 247-8, 249, 256, 259-60

see also social service

Wales 111, 153, 154, 185, 229, 230, 249

Wallace, Alfred Russel 80-1

Webb, Sidney and Beatrice 17-18, 22, $26,47,59,125,129,135$

Weight of trainees 48, 65, 107, 108, $166-7,214-15$

Welshman, J. 143

Whitby 150, 153, 196

Women 37, 43, 45, 50, 60, 62-3, 68-9, $71,80,83-4,86,90-3,110$, 115, 173-94, 200-3, 212, 214, $248,252-3$

Woodcraft movement 208-11

work routine $34-5,36-7,40-1,70$, 157-9, 166, 183, 185, 205, 209, 211

Workers' Educational Association 210, 249-50

workhouse 2, 9, 10-12, 39, 41, 49-50, $57-8,68,79,174,223$

Wright, Samson 212

\section{Young Men's Christian Association} 115,158

Zionists 200, 211-13

Zweiniger-Bargielowska, Ina 57 